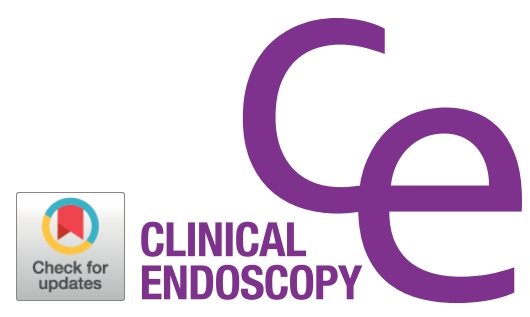

\title{
Diagnosis of Pancreatic Neuroendocrine Tumors
}

\author{
Dong Wook Lee ${ }^{1}$, Michelle Kang Kim² and Ho Gak Kim \\ ${ }^{1}$ Department of Internal Medicine, Daegu Catholic University School of Medicine, Daegu, Korea, ${ }^{2}$ Division of Gastroenterology, Department \\ of Medicine, Icahn School of Medicine, The Mount Sinai Medical Center, New York, NY, USA
}

Pancreatic neuroendocrine tumors (PNETs) are relatively rare; however, the incidence has increased over the last few decades. They are classified as functional or non-functional tumors according to the presence of associated clinical symptoms. The majority are nonfunctional tumors. For classification and staging, the World Health Organization 2010 classification system is the most commonly accepted. Chromogranin A is the most sensitive marker but has insufficient specificity. In general, PNETs are hypervascular tumors, and multiphasic contrast-enhanced computed tomography is considered the first choice for imaging study. Multiphasic magnetic resonance imaging can detect PNETs smaller than $2 \mathrm{~cm}$ and small liver metastasis compared with other modalities. Somatostatin receptor scintigraphy is often used in cases where functional PNETs are suspected. Positron emission tomography (PET) scan with 18F-fluorodeoxyglucose cannot visualize PNETs, but PET with 68-Ga DOTATATE can. Endoscopic ultrasonography can characterize smaller PNETs using contrast and confirm histology through fine needle aspiration or biopsy. In this article, we review the characteristics of grading systems and diagnostic modalities commonly used for PNETs. Clin Endosc 2017;50:537-545

Key Words: Pancreatic neuroendocrine tumor; World Health Organization classification; Computed tomography; Magnetic resonance imaging; Endoscopic ultrasonography

\section{INTRODUCTION}

Neuroendocrine tumors (NETs) can arise from different organs. The lungs, gastrointestinal (GI) tract, pancreas are common sites. GI and pancreatic NETs (PNETs) are histologically classified under the same category; however, PNETs have different clinical features from GI NETs and, thus, should be considered separately.

PNETs are relatively rare and comprise only $1 \%-2 \%$ of pancreatic tumors. ${ }^{1}$ With the recent advances in imaging technology and understanding of these tumors, the diagnosis of asymptomatic and symptomatic PNETs has improved.

PNETs are divided into functional and non-functional tumors. About $90 \%$ of PNETs are classified as non-functional

Received: August 17, 2017 Accepted: October 15, 2017

Correspondence: Ho Gak Kim

Department of Internal Medicine, Daegu Catholic University School of Medicine, 33 Duryugongwon-ro 17-gil, Nam-gu, Daegu 42472, Korea

Tel: +82-53-650-4041, Fax: +82-53-621-4487, E-mail: hgkim@cu.ac.kr

(cc) This is an Open Access article distributed under the terms of the Creative Commons Attribution Non-Commercial License (http://creativecommons.org/ licenses/by-nc/3.0) which permits unrestricted non-commercial use, distribution, and reproduction in any medium, provided the original work is properly cited. tumors, and the remaining $10 \%$ as functional tumors. ${ }^{2}$ Functional tumors secrete particular hormones or peptides, such as insulin, gastrin, vasoactive intestinal peptide (VIP), glucagon, and somatostatin.

In this review, we summarize the classification, grading, and diagnosis of PNETs, with a focus on imaging studies.

\section{CLASSIFICATION AND GRADING SYSTEM}

The classification and grading system of PNETs is varied and has been modified for the last 15 years. According to the World Health Organization (WHO) 2004 classification, PNETs can be classified into four groups: well-differentiated endocrine tumor, benign; well-differentiated endocrine tumor with uncertain behavior; well-differentiated endocrine tumor carcinoma; and poorly-differentiated endocrine tumor carcinoma. ${ }^{3}$ However, this classification is not widely accepted in practice. In 2010, a new WHO classification for GI NETs and PNETs was proposed (Table 1). ${ }^{4}$

To date, three systems of grading or staging for PNETs, 
which were developed by the European Neuroendocrine Tumor Society (ENETS), WHO 2010, and American Joint Committee on Cancer (AJCC), are widely accepted. ENETS proposed a staging system based on tumor, node, metastasis (TNM) classification (Table 2). ${ }^{5}$ The WHO 2010 classification system classified PNETs into two categories based on mitotic count and Ki-67 index: well-differentiated and poorly-differentiated tumors. ${ }^{4}$ Well-differentiated tumors are referred to as NETs and further divided into low grade (G1) and intermediate grade (G2) tumors. Poorly-differentiated tumors are referred to as neuroendocrine carcinomas and high grade (G3) tumors (Table 1). The WHO 2010 classification system is useful in predicting the survival rate of patients with PNETs and widely used as a guideline for the treatment of PNETs. ${ }^{6}$ In addition, the AJCC staging system is based on the TNM staging system for pancreatic adenocarcinoma (Table 2).

For PNETs, 50\%-100\% of cases are malignant, except for insulinoma, wherein only $10 \%$ of cases are malignant. ${ }^{1}$ The liver is the most common site of metastasis, and the size of the primary tumor is an independent predictor of the development of liver metastasis. ${ }^{8}$

\section{DIAGNOSIS}

\section{History and clinical manifestation}

Detailed history taking and physical examination are also important for the diagnosis of patients with PNETs. However, the majority of PNETs are non-functional tumors and asymptomatic upon diagnosis and are incidentally found on imaging study. Patients with non-functional PNETs sometimes present with symptoms or signs by mass effect or metastasis, including jaundice caused by extrahepatic bile duct occlusion, acute pancreatitis resulting from main pancreatic duct compression, abdominal pain, and palpable abdominal mass.

In the case of functional PNETs, the specific symptoms and signs are caused by hormones or peptides they release. The most common functional PNETs are insulinomas and gastrinomas, followed by less common VIPomas, glucagonomas, and somatostatinomas (Table 3).

Insulinomas are the most common functional PNETs, which comprise $35 \%-40 \%,{ }^{2}$ and patients with PNETs present with hypoglycemia due to episodic hyperinsulinemia. The classical diagnosis of insulinoma meets the criteria of Whipple’s triad: (1) hypoglycemia (plasma glucose $<50 \mathrm{mg} / \mathrm{dL}$ ), (2) symptoms of hypoglycemia (weakness, sweating, palpitations,

Table 1. WHO 2010 Classification and Suggested Grading

\begin{tabular}{lllcc}
\hline Classification & & Grade & $\begin{array}{c}\text { Mitotic count } \\
\text { (per 10 HPF) }\end{array}$ & \multicolumn{2}{c}{$\begin{array}{c}\text { Ki-67 index } \\
\text { (\%) }\end{array}$} & $<2$ \\
\hline Well-differentiated & NET & G1: low grade & $2-20$ & $3-20$ \\
& & G2: intermediate grade & $>20$ & $>20$ \\
Poorly-differentiated & NEC & G3: high grade & & $<2$ \\
\hline
\end{tabular}

WHO, World Health Organization; HPF, high power field; NET, neuroendocrine tumor; NEC, neuroendocrine carcinoma.

Table 2. ENETS and AJCC TNM Classifications and Stage of PNETs

\begin{tabular}{|c|c|c|c|c|c|}
\hline & & \multicolumn{2}{|c|}{ ENETS TNM } & \multicolumn{2}{|c|}{ AJCC TNM } \\
\hline \multirow[t]{4}{*}{ TNM } & $\mathrm{T} 1$ & \multicolumn{2}{|c|}{ Confined to pancreas, $<2 \mathrm{~cm}$} & \multicolumn{2}{|c|}{ Confined to pancreas, $<2 \mathrm{~cm}$} \\
\hline & $\mathrm{T} 2$ & \multicolumn{2}{|c|}{ Confined to pancreas, $2-4 \mathrm{~cm}$} & \multicolumn{2}{|c|}{ Confined to pancreas, $>2 \mathrm{~cm}$} \\
\hline & T3 & \multicolumn{2}{|c|}{$\begin{array}{l}\text { Confined to pancreas, }>4 \mathrm{~cm} \text {, or invasion of duode- } \\
\text { num or bile duct }\end{array}$} & \multicolumn{2}{|c|}{$\begin{array}{l}\text { Peripancreatic spread, but without major vascular inva- } \\
\text { sion (celiac trunk, SMA) }\end{array}$} \\
\hline & $\mathrm{T} 4$ & \multicolumn{2}{|c|}{ Invasion of adjacent organs or major vessels } & \multicolumn{2}{|c|}{ Major vascular invasion } \\
\hline \multirow[t]{7}{*}{ Stage } & & \multirow[t]{2}{*}{ I } & \multirow[t]{2}{*}{ T1 N0 M0 } & IA & T1 N0 M0 \\
\hline & & & & IB & T2 N0 M0 \\
\hline & & IIA & T2 N0 M0 & IIA & T3 N0 M0 \\
\hline & & IIB & T3 N0 M0 & IIB & T1-3 N1 M0 \\
\hline & & IIIA & T4 N0 M0 & III & T4 Any N M0 \\
\hline & & IIIB & Any T N1 M0 & & \\
\hline & & IV & Any T Any N M1 & IV & Any T Any N M1 \\
\hline
\end{tabular}

ENETS, European Neuroendocrine Tumor Society; AJCC, American Joint Committee on Cancer; TNM, tumor, node, metastasis; PNETs, pancreatic neuroendocrine tumors, SMA, superior mesenteric artery. 
Table 3. Symptoms and Signs of Functional PNETs, and Their Incidence

\begin{tabular}{|c|c|c|c|}
\hline Name of tumor & $\begin{array}{l}\text { Secreting hormone or } \\
\text { peptide }\end{array}$ & Symptoms and signs & $\begin{array}{c}\text { Percentage in } \\
\text { functional PNETs }\end{array}$ \\
\hline Insulinoma & Insulin & Episodes hypoglycemic symptoms & $35 \%-40 \%$ \\
\hline $\begin{array}{l}\text { Gastrinoma (Zollinger-El- } \\
\text { lison syndrome) }\end{array}$ & Gastrin & Refractory peptic ulcer at stomach, duodenum, diarrhea & $16 \%-30 \%$ \\
\hline Glucagonoma & Glucagon & Dermatitis with migratory necrotic erythema, diarrhea, DVT & $<10 \%$ \\
\hline VIPoma & VIP & Profuse watery diarrhea, hypokelemia & $<10 \%$ \\
\hline Somatostatinoma & Somatostatin & $\begin{array}{l}\text { Subtle symptoms including diabetes mellitus, biliary stone, ste- } \\
\text { atorrhea, weight loss }\end{array}$ & $<5 \%$ \\
\hline
\end{tabular}

PNETs, pancreatic neuroendocrine tumors; VIP, vasoactive intestinal peptide; DVT, deep vein thrombosis.

confusion), and (3) prompt relief of symptoms after administration of glucose. 9 The symptoms of gastrinomas result from hypersecretion of gastrin, causing refractory peptic ulcer in the stomach and duodenum and secretory diarrhea, referred to as Zollinger-Ellison syndrome. The most common symptom of glucagonomas is dermatitis with migratory necrotic erythema. Other symptoms include glucose intolerance, diarrhea, and deep vein thrombosis. Large volume secretory watery diarrhea and hypokalemia are common symptoms of VIPomas. Somatostatinomas are extremely rare and cause very subtle symptoms such as abdominal pain and weight loss (Table 3).

When specific symptoms of functional PNETs are observed, the evaluation of imaging study and specific hormone or peptide level should be carried out cautiously.

\section{Laboratory evaluation}

The evaluation of hormones or peptides, including insulin, glucagon, gastric, VIP, and somatostatin, is essential for the diagnosis of functional PNETs when signs or symptoms are present (Table 3). The serum level of insulin and C-peptide along with glucose during prolonged fasting (up to $72 \mathrm{~h}$ ) is useful for the diagnosis of insulinoma if symptoms of hypoglycemia are present or plasma glucose level is below $49 \mathrm{mg} /$ dL. Normally, insulin levels are decreased during hypoglycemia, but in patients with insulin-secreting tumors, they do not decrease and C-peptide levels are elevated during hypoglycemia. Proinsulin levels are also elevated in insulinoma (Table 4). ${ }^{10,11}$

When gastrinoma is suspected, fasting serum gastrin levels should be evaluated. A serum gastrin level that is 10 times greater than the upper limit of the normal range along with a gastric $\mathrm{pH}<2$ is diagnostic of gastrinoma. ${ }^{9,12}$ If gastric $\mathrm{pH}$ is $>2$, secondary hypergastrinemia is suspected, which can occur with proton pump inhibitor administration, atrophic gastritis, and post-vagotomy state.

PNETs, including insulinomas, gastrinomas, somatostatinomas, and even non-functional tumors, can be associated with
Table 4. Biochemical Diagnosis of Insulinoma at The Time of Hypoglycemia During Prolonged Fasting (up to 72 hours)

\begin{tabular}{lc}
\hline Biochemical parameter & Suspecting insulinoma \\
\hline Insulin $(\mathrm{mIU} / \mathrm{L})$ & $\geq 5$ \\
Glucose $(\mathrm{mg} / \mathrm{dL})$ & $<40$ \\
C-peptide $(\mathrm{ng} / \mathrm{mL})$ & $>0.6$ \\
Proinsulin $(\mathrm{pmol} / \mathrm{L})$ & $\geq 20$ (or $>25 \%$ of immunoreactive \\
& insulin) \\
\hline
\end{tabular}

multiple endocrine neoplasia type 1 (MEN 1), which includes pituitary adenoma, parathyroid hyperplasia, and duodenal gastrinoma. When MEN 1 is suspected, serum prolactin, parathyroid hormone, and gastrin levels should be evaluated additionally.

In case of dermatitis, such as necrolytic migratory erythe$\mathrm{ma}$, the diagnosis of glucagonoma requires increased plasma glucagon level greater than 10-fold (normal glucagon $<50 \mathrm{pg} /$ $\mathrm{mL}){ }^{11,13}$ The diagnosis of VIPoma and somatostatinoma is confirmed through the fasting level of VIP and somatostatin in patients with suspected symptoms.

Several tumor markers for functional and non-functional PNETs have been previously studied. The most widely used markers are chromogranin A ( $\mathrm{CgA})$, neuron-specific enolase (NSE), and pancreatic polypeptide. $\mathrm{CgA}$, a protein that is stored in the secretory granules of different NETs, is a highly sensitive marker for the diagnosis of NETs. ${ }^{14}$ ELISA kits for CgA are commercially available in the USA and Europe, and serum enzyme immunoassay study for $\mathrm{CgA}$ is available in South Korea. Elevated CgA level is correlated with tumor burden and metastasis of well-differentiated NETs rather than poorly-differentiated NETs. ${ }^{15}$ Moreover, it may be useful in assessing responses to therapy and following up the tumor. ${ }^{16}$ However, the CgA level of healthy participants and those with NETs varies day by day and is influenced by food intake. The most common cause of elevated $\mathrm{CgA}$ is the use of proton pump inhibitors, and it can also be elevated with renal and hepatic insufficiency. Therefore, CgA is not specific for the diagnosis of NETs. ${ }^{17,18}$ 
NSE is an enzyme released after neuronal damage caused by different conditions, such as brain ischemia. In addition, this enzyme is identified in the cytoplasm of NET cells. The sensitivity of NSE as a tumor marker for NETs is as low as $30 \%-40 \%$, but the specificity is almost $100 \%{ }^{19}$ Using a combination of CgA and NSE improves the sensitivity for NETs. ${ }^{20}$

\section{Imaging}

\section{Overview}

Various imaging modalities to stage and localize PNETs are used in the clinical setting, including computed tomography (CT), magnetic resonance imaging (MRI), Somatostatin receptor scintigraphy using somatostatin receptor analogue octreotide (OctreoScan ${ }^{\circledR}$ ), positron emission tomography (PET), endoscopic ultrasonography (EUS), and selective angiography. In the past, celiac or mesenteric arteriography was important in confirming the mass of GI NETs and PNETs because NETs are hypervascular tumors. With the advance- ment in contrast-enhancement and multiphasic techniques, contrast-enhanced CT and MRI has replaced angiography. However, angiography with selective arterial stimulation and venous sampling for specific hormones may be used for localization of functional tumors, which cannot be visualized with imaging studies. ${ }^{21}$ The introduction and development of EUS in the last couple of decades opened a new era of diagnosis and treatment of PNETs.

\section{Computed tomography}

CT is the most common initial imaging study in the evaluation of patients with hormonal syndrome suspected with functional PNETs. Moreover, it is recommended for the evaluation of patients with mass on other imaging studies, although the patients have no evidence of symptoms or signs of functional PNETs.

Helical (spiral) triple-phase contrast-enhanced CT is the best option for the assessment of highly vascularized PNETs and liver metastasis. PNETs and metastatic mass are typically visualized during early arterial phase with washout during
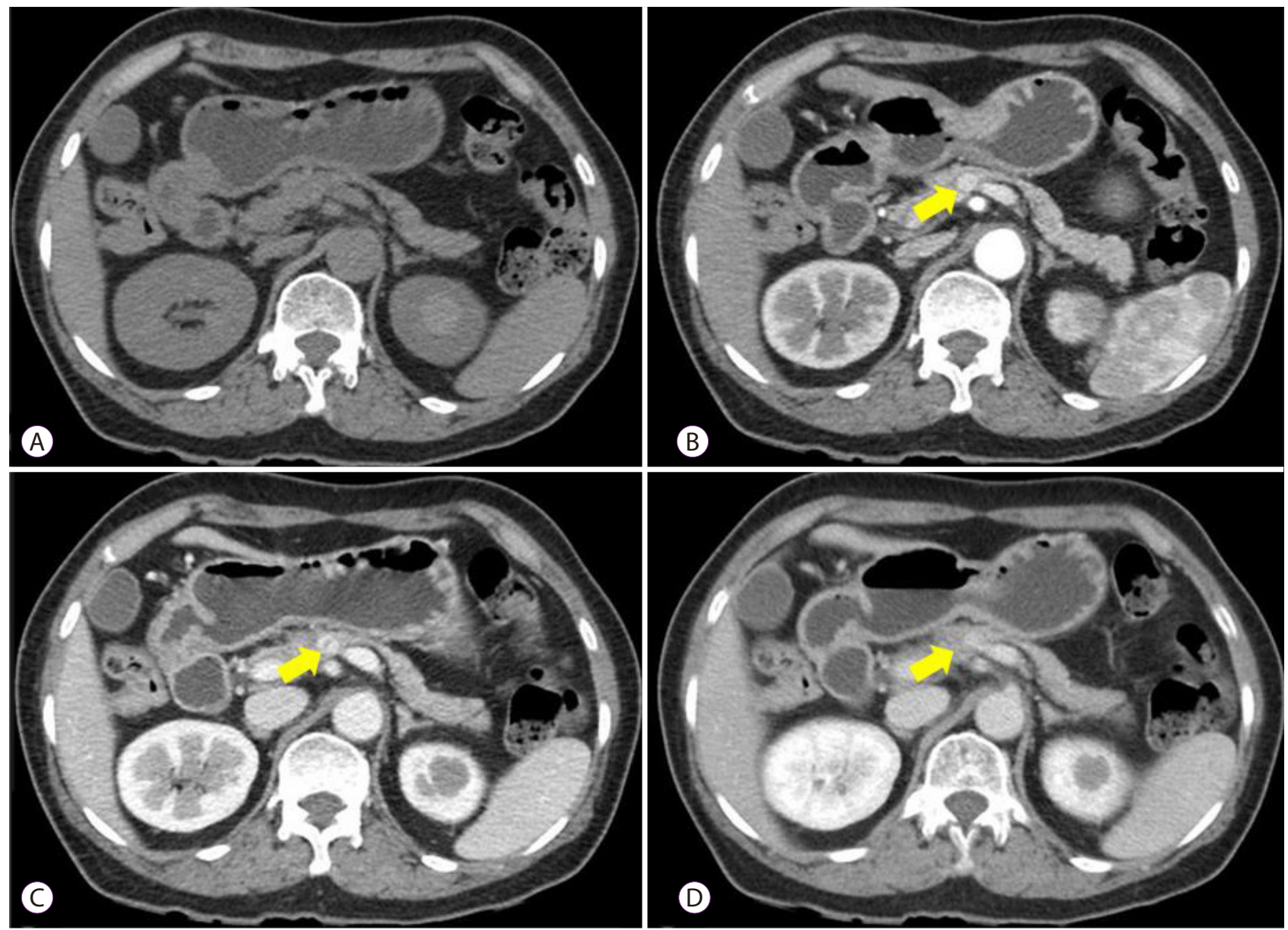

Fig. 1. Pancreatic neuroendocrine tumor in a 69-year-old female. A $1.0 \mathrm{~cm}$ size, round, hyperattenuating mass (yellow arrow) was seen in the pancreas body. The mass was not observed in non-enhanced phase (A), but highly enhanced in the early arterial phase (B). Contrast was washed out in the late arterial (C) and venous phases (D). 
portal venous phase..$^{22}$ In contrast-enhanced CT, PNETs appear as a round, solid, and hypervascular mass (Fig. 1). However, about $10 \%$ present as cystic lesions with smooth margin and peripheral enhancement (Fig. 2). 2,23 The mass is usually located within the parenchyma of the pancreas and rarely obstructs the pancreatic duct, but sometimes the mass is exophytic to the pancreas capsule or obstructs the bile and pancreatic ducts.

Contrast-enhanced CT scans are highly accurate in detecting PNETs larger than $2 \mathrm{~cm}$ and have a sensitivity range of $63 \%-82 \%$ and specificity range of $83 \%-100 \%{ }^{24,25}$ The sensitivity is decreased in tumors smaller than $2 \mathrm{~cm}$. However, symptomatic non-functional and functional tumors with hormone syndrome are usually larger than $2-3 \mathrm{~cm}$ at presentation. ${ }^{17,26}$ CT scan also has another advantage: they can define the resectability of the tumor and detect the presence of liver and intraabdominal metastases.
Multiphasic contrast-enhanced CT is considered the imaging study of choice for detecting and staging PNETs.

\section{Magnetic resonance imaging}

Currently, with the advent of new technology in MRI sequences, PNETs are well visualized on MRI. They typically show low signal intensity on T1-weighted images and high signal intensity on T2-weighted images (Fig. 3). Similar to CT scan images, early arterial phase image is the best for detecting hypervascular PNETs and small metastasis on gadolinium contrast-enhanced MRI. ${ }^{27}$ The sensitivity of MRI is over $85 \%$, and its specificity is over $75 \%{ }^{24,28}$

Multiphasic MRI can detect PNETs that are less than $2 \mathrm{~cm}$ and small liver metastasis compared with CT scan. ${ }^{29}$ However, MRI sequence has certain limitations: it produces respiratory motion artifacts and cannot be used in patients with cardiac pacemaker or implanted metal devices. Recently, a new MRI
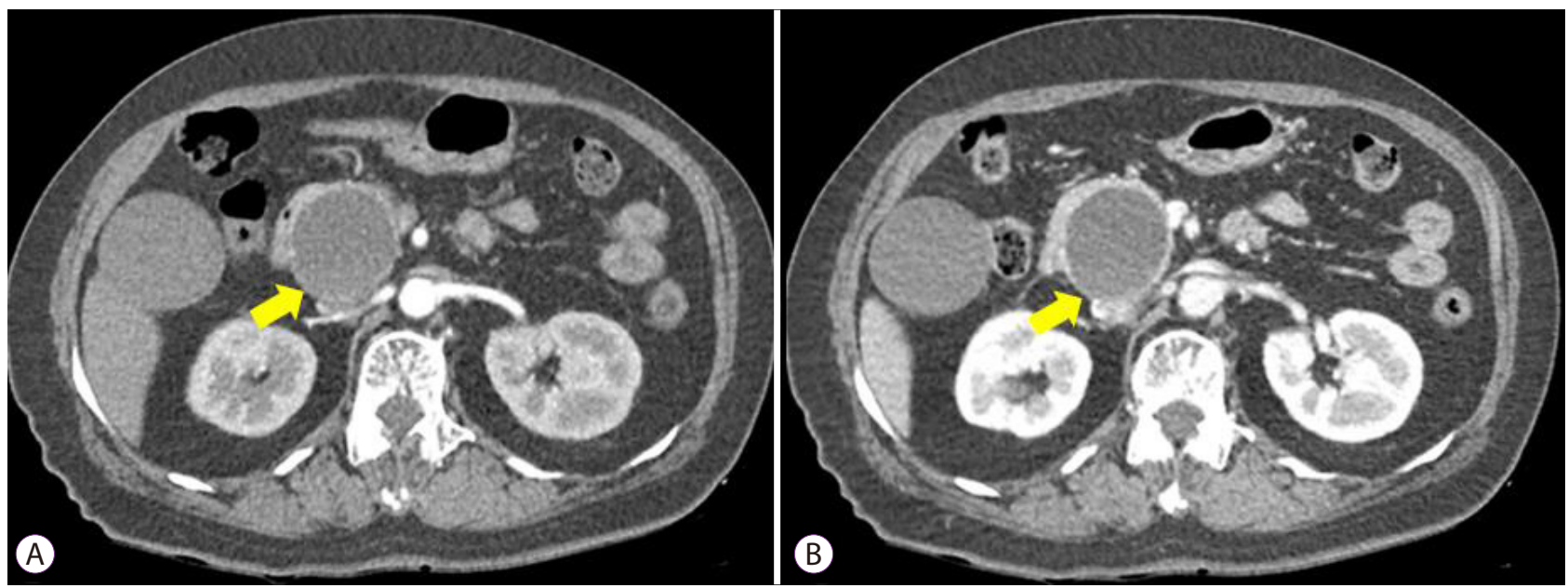

Fig. 2. Pancreatic neuroendocrine tumor with cystic degeneration in a 70-year-old female. $A 4.8 \mathrm{~cm}$ size, round, cystic mass (yellow arrow) was seen in the pancreas head. Peripheral enhancement was observed in the early arterial $(A)$ and late arterial phases (B).
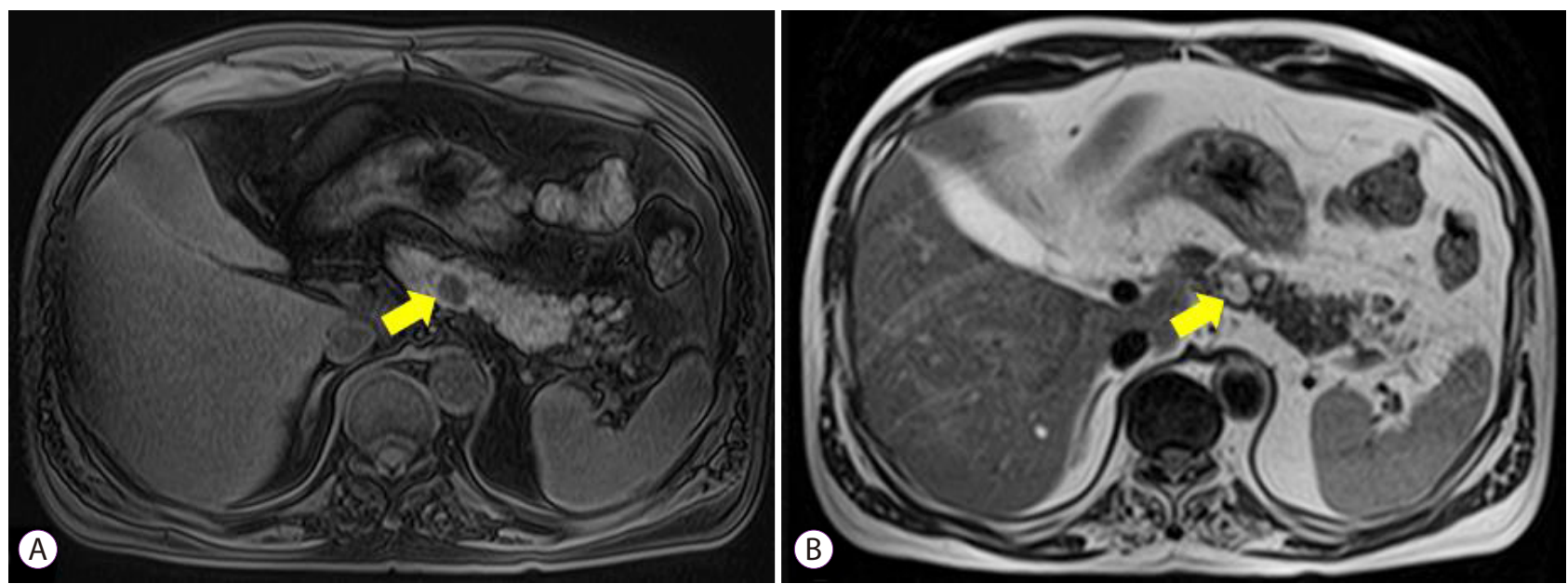

Fig. 3. Pancreatic neuroendocrine tumor in a 59-year-old male. A $1.5 \mathrm{~cm}$ size, round mass (yellow arrow) was seen in the pancreas body. The mass demonstrates low signal intensity relative to the normal high signal intensity of the pancreas on T1-weighted image (A) and high signal intensity on T2-weighted image (B). 
sequence system is being developed to overcome these limitations.

Compared with other imaging modalities, MRI has been suggested to be more sensitive in detecting small tumors and liver metastasis ${ }^{29}$ and is a second-line method of imaging for the localization of PNETs. ${ }^{30,31}$

\section{Somatostatin receptor scintigraphy}

Many PNETs, including non-functional tumors except insulinomas, express high levels of somatostatin receptors. Radiolabeled somatostatin analog octreotide (111-In pentetreotide), also called OctreoScan, can induce uptake in PNETs. This method has a positive uptake for visualizing gastrinomas, glucagonomas, and even non-functional tumors. ${ }^{32,33}$ It can be particularly helpful when glucagonoma is located outside of the pancreas. ${ }^{24}$ The sensitivity of 111-In pentetreotide scintigraphy for the detection of gastrinomas, VIPomas, glucagonomas, and clinically non-functioning lesions is $75 \%-100 \%{ }^{34}$ Insulinomas and poorly-differentiated PNETs are usually undetected with somatostatin receptor scintigraphy because they express low levels of somatostatin receptors. ${ }^{33,35}$

Somatostatin receptor scintigraphy is often used when functional PNETs are suspected but tumors are not localized on cross-sectional images. It also has advantage before the decision of administration of octretide analog for the treatment of well-differentiated PNETs and targeting radiation therapy using radiolabeled somatostatin analogs.

\section{Positron emission tomography}

Most PNETs cannot be visualized through PET scan with 18F-fluorodeoxyglucose (FDG) because they are well differentiated and have low metabolic activity. However, poorly-differentiated PNETs have increased metabolic rate with FDG uptake and can be detected with PET scan. FDG activity on
PET scan is correlated with tumor progression and increased mortality. ${ }^{36,37}$

A new PET tracer for NETs, 68-Ga DOTATATE, was recently approved by the Food and Drug Administration (FDA), and numerous studies showed that it has greater sensitivity than 111-In pentetreotide scintigraphy in detecting small lesions. ${ }^{38,39}$ A combination of PET with 68-Ga DOTATATE and high-resolution CT may be preferred over conventional somatostatin receptor scintigraphy if it is available.

\section{Endoscopic ultrasonography}

EUS has become a very useful imaging modality to evaluate pancreatic lesions. With high-frequency transducer, it provides a high-resolution image of the pancreas. A recent study on 56 patients with PNETs showed that EUS is superior to multidetector CT for the detection of PNETs even though CT technology has improved. ${ }^{40}$ In the same study on 231 patients with PNETs, lesions smaller than $2 \mathrm{~cm}$ and insulinomas were usually missed by CT. ${ }^{40}$

Based on EUS, PNETs are typically well-defined hypoechoic homogenous lesions (Fig. 4). Majority of PNETs are solid but occasionally cystic or have a cystic portion on the solid mass (Fig. 5).

EUS has advantages as compared to other imaging modalities in several aspects. First, it can detect PNETs that are smaller than 2-3 cm in diameter, which were not often detected by $\mathrm{CT}^{40}$ In a systematic review of 17 cohort studies, EUS identified PNETs in $97 \%$ of cases. Among them, $28 \%$ of EUS-detected PNETs were not detected by $\mathrm{CT}^{41}{ }^{11}$ Second, peripancreatic abnormalities caused by PNETs can be examined during EUS. The enlargement of local lymph node and vascular invasion by mass can be detected through EUS. EUS is also useful in identifying gastrinomas that are located in the duodenal wall and small insulinomas that were not previously detected by
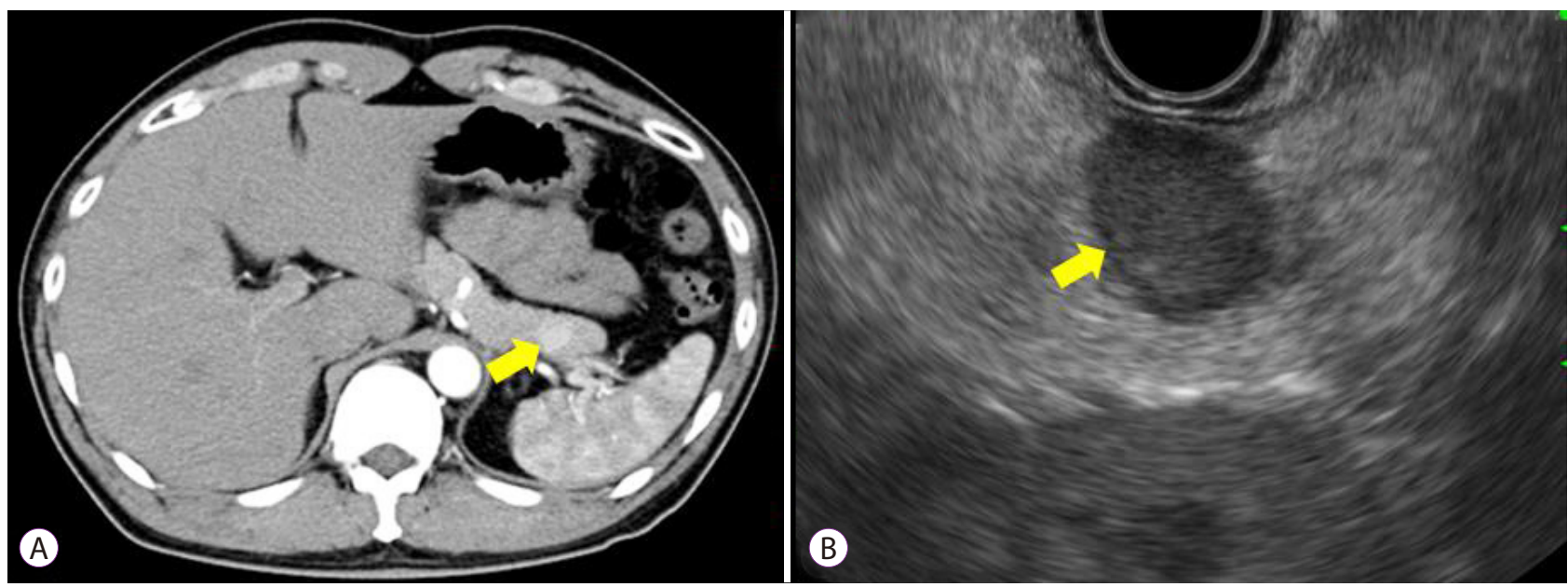

Fig. 4. Pancreatic neuroendocrine tumor in a 47-year-old female. A $1.2 \mathrm{~cm}$ size, round, hyperattenuating mass (yellow arrow) was seen in the pancreas tail on computed tomography (A). The mass was well defined and displayed a hypoechoic, homogeneous pattern on endoscopic ultrasonography (B). 
CT, somatostatin receptor scintigraphy, or 18F-FDG PET because of the low expression of somatostatin receptors. Third, tissue acquisition through fine needle aspiration or fine needle biopsy with linear array EUS can provide a histological confirmation of PNETs before surgery. Lastly, EUS has an important role in tattooing small lesions for easier intraoperative identification through the injection of carbon-based ink into the mass. ${ }^{42,43}$ Moreover, it can be used for the local treatment of pancreatic tumors. EUS-guided ethanol ablation of PNETs has high success rate and low rate of procedure-related complications. ${ }^{44,45}$ However, the volume and optimal concentration of ethanol that should be injected and techniques of EUS-guided injection should be further refined. ${ }^{45}$

Contrast-enhanced EUS is helpful in characterizing small PNETs, which are incidentally found on other imaging modalities. ${ }^{46}$ Over $90 \%$ of PNETs showed hypoechogenicity in
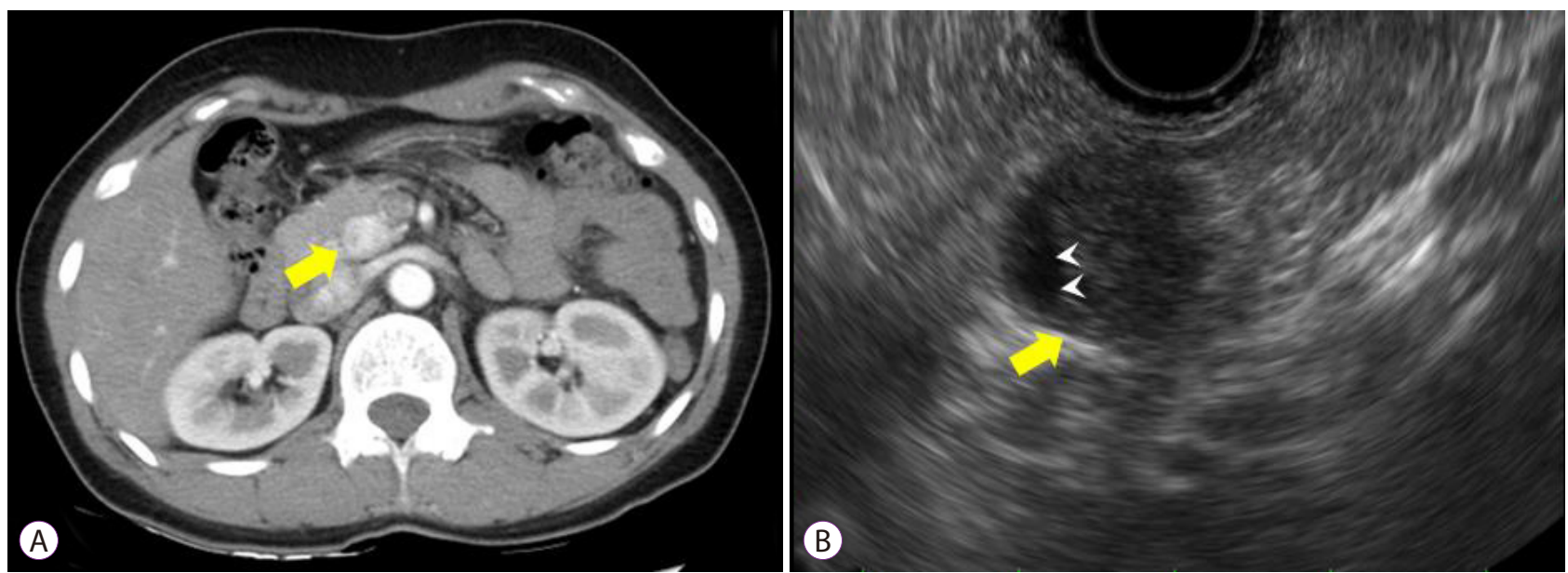

Fig. 5. Pancreatic neuroendocrine tumor in a 45-year-old female. A $2.0 \mathrm{~cm}$ size, round, hyperattenuating mass (yellow arrow) was seen in the pancreas head on computed tomography (A). The mass was also observed on endoscopic ultrasonography with internal cystic portion (arrow head) (B).
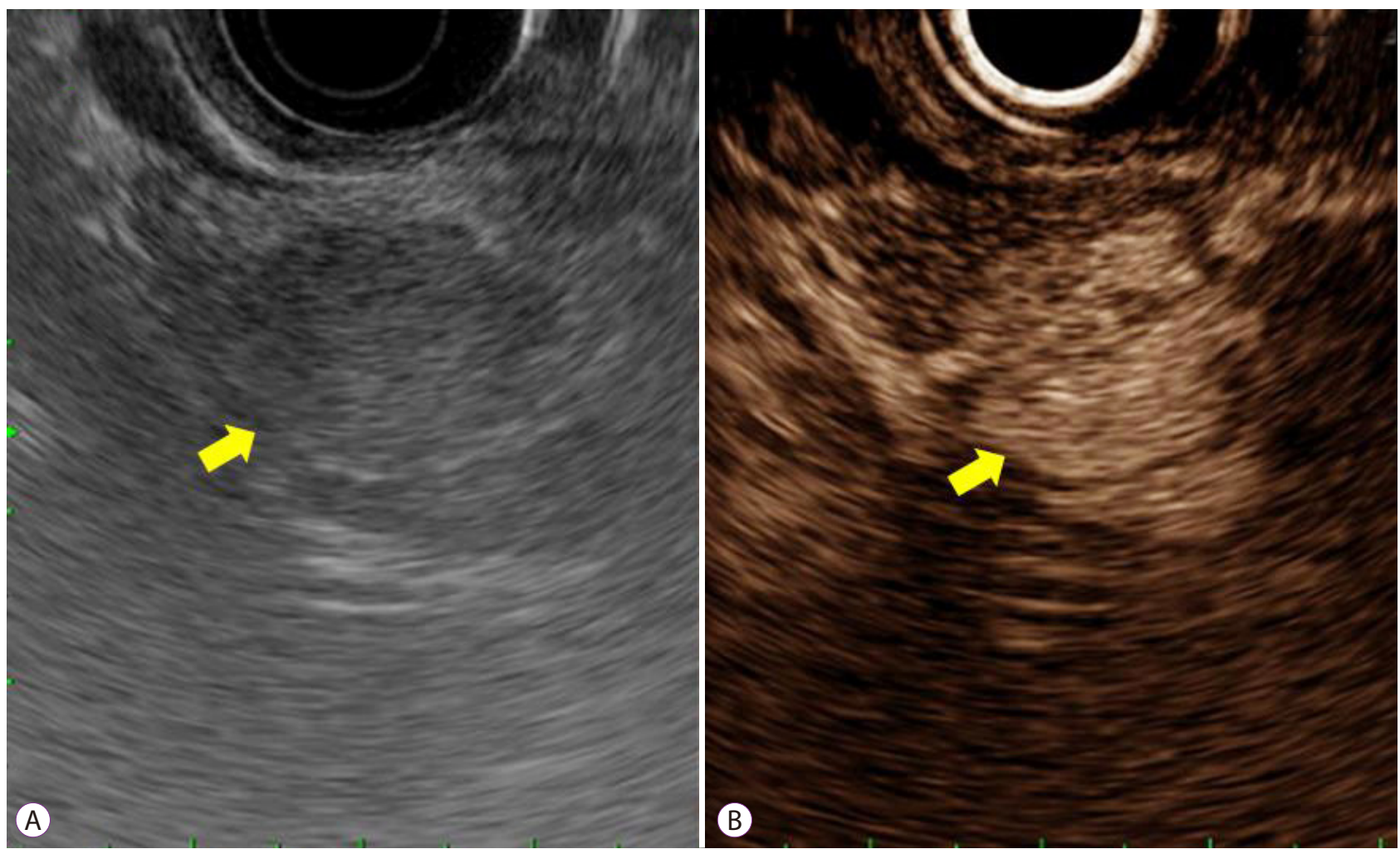

Fig. 6. Pancreatic neuroendocrine tumor in a 42-year-old male. A $1.5 \mathrm{~cm}$ size, round, hypoechoic, homogeneous mass (yellow arrow) was seen in the pancreas tail on conventional endoscopic ultrasonography (A). After contrast injection, hyperenhancement was observed in the mass on contrast-enhanced endoscopic ultrasonography (B). 
B-mode and hyperenhancement after the injection of contrast agent in contrast-enhanced EUS (Fig. 6). In a study on 156 patients with suspected small pancreatic tumors by contrast-enhanced EUS, $75 \%$ of hypervascular lesions were NETs. ${ }^{47}$

However, EUS has several limitations: it is dependent on an endoscopist, which may create inconsistent results, and the pancreas tail mass is poorly visualized because of inadequate ultrasonic window in some cases.

\section{CONCLUSIONS}

Among pancreatic neoplasm, PNETs are rare tumor. However there incidence is increasing because of the advancement of imaging technology and increased opportunities for checking pancreatic diseases. The majority of PNETs are non-functional tumors and create symptoms due to the mass itself. Some patients present with symptoms secondary to hormone overproduction from functional PNETs. Insulinomas are the most common functional PNETs.

For classification and staging, the ENETS, WHO 2010, and AJCC systems are used. Among them, the WHO 2010 classification system is the most commonly accepted. PNETs are classified into two categories based on mitotic count and Ki-67 index (well-differentiated and poorly-differentiated tumors). Malignant potential of PNETs is relatively common and size of the primary tumor and liver metastasis are predictors of malignant potential.

A detailed history taking of symptoms caused by mass effect and hormone overproduction is essential for the diagnosis of PNETs. Laboratory tests for functional PNETs include hormone levels for specific symptoms and tumor markers, such as CgA and NSE. CgA is the most sensitive marker and is correlated with tumor burden but has limited specificity.

For localization and staging of PNETs, multiphasic contrast-enhanced CT is considered the imaging study of choice. MRI has been suggested to be more sensitive in detecting small tumors and liver metastasis than other modalities. Thus, MRI is the second-line method of imaging for the localization of PNETs, which are not confirmed with other modalities. somatostatin receptor scintigraphy is often used when functional PNETs, except insulinomas, are suspected clinically but tumors are not localized on cross-sectional images. PNETs cannot be visualized on PET scan with 18F-FDG because a majority of them are well differentiated. However, PET with 68-Ga DOTATATE has been approved recently by the FDA and has improved sensitivity. EUS provides high-resolution ultrasonic images of the pancreas and can detect small tumors less than 2-3 cm. Its sensitivity is equal to or superior to multidetector CT or MRI for the detection of PNETs. Other ben- efits of EUS include the detection of lymph node involvement and vascular invasion and the possibility of tissue acquisition through fine needle aspiration or biopsy. Contrast-enhanced EUS is helpful in categorizing small hypervascular PNETs, which are incidentally found on other imaging modalities.

Early detection through advanced imaging modalities and proper classification and staging with the recently updated classification system are important in providing prognostic information and decision for the management of PNETs.

Conflicts of Interest

The authors have no financial conflicts of interest.

\section{REFERENCES}

1. Lawrence B, Gustafsson BI, Chan A, Svejda B, Kidd M, Modlin IM. The epidemiology of gastroenteropancreatic neuroendocrine tumors. Endocrinol Metab Clin North Am 2011;40:1-18, vii.

2. McKenna LR, Edil BH. Update on pancreatic neuroendocrine tumors. Gland Surg 2014;3:258-275.

3. Solcia E, Sessa F, Rindi G, et al. Classification and histogenesis of gastroenteropancreatic endocrine tumours. Eur J Clin Invest 1990;20 Suppl 1:S72-S81.

4. Rindi G, Petrone G, Inzani F. The 2010 WHO classification of digestive neuroendocrine neoplasms: a critical appraisal four years after its introduction. Endocr Pathol 2014;25:186-192.

5. Rindi G, Klöppel G, Alhman H, et al. TNM staging of foregut (neuro) endocrine tumors: a consensus proposal including a grading system. Virchows Arch 2006;449:395-401.

6. Ellison TA, Wolfgang CL, Shi C, et al. A single institution's 26-year experience with nonfunctional pancreatic neuroendocrine tumors: a validation of current staging systems and a new prognostic nomogram. Ann Surg 2014;259:204-212.

7. Strosberg JR, Cheema A, Weber J, Han G, Coppola D, Kvols LK. Prognostic validity of a novel American joint committee on cancer staging classification for pancreatic neuroendocrine tumors. J Clin Oncol 2011;29:3044-3049.

8. Riihimäki M, Hemminki A, Sundquist K, Sundquist J, Hemminki K. The epidemiology of metastases in neuroendocrine tumors. Int J Cancer 2016;139:2679-2686.

9. Metz DC, Jensen RT. Gastrointestinal neuroendocrine tumors: pancreatic endocrine tumors. Gastroenterology 2008;135:1469-1492.

10. Vezzosi D, Bennet A, Fauvel J, Caron P. Insulin, C-peptide and proinsulin for the biochemical diagnosis of hypoglycaemia related to endogenous hyperinsulinism. Eur J Endocrinol 2007;157:75-83.

11. van Beek AP, de Haas ER, van Vloten WA, Lips CJ, Roijers JF, Canninga-van Dijk MR. The glucagonoma syndrome and necrolytic migratory erythema: a clinical review. Eur J Endocrinol 2004;151:531-537.

12. Berna MJ, Hoffmann KM, Serrano J, Gibril F, Jensen RT. Serum gastrin in Zollinger-Ellison syndrome: I. prospective study of fasting serum gastrin in 309 patients from the national institutes of health and comparison with 2229 cases from the literature. Medicine (Baltimore) 2006;85:295-330.

13. Wermers RA, Fatourechi V, Wynne AG, Kvols LK, Lloyd RV. The glucagonoma syndrome. Clinical and pathologic features in 21 patients. Medicine (Baltimore) 1996;75:53-63.

14. Campana D, Nori F, Piscitelli L, et al. Chromogranin A: is it a useful marker of neuroendocrine tumors? J Clin Oncol 2007;25:1967-1973.

15. Modlin IM, Gustafsson BI, Moss SF, Pavel M, Tsolakis AV, Kidd M. Chromogranin A--biological function and clinical utility in neuro endocrine tumor disease. Ann Surg Oncol 2010;17:2427-2443. 
16. Nehar D, Lombard-Bohas C, Olivieri S, et al. Interest of chromogranin A for diagnosis and follow-up of endocrine tumours. Clin Endocrinol (Oxf) 2004;60:644-652

17. Paik WH, Ryu JK, Song BJ, et al. Clinical usefulness of plasma chromogranin a in pancreatic neuroendocrine neoplasm. J Korean Med Sci 2013;28:750-754.

18. Vinik AI, Silva MP, Woltering EA, Go VL, Warner R, Caplin M. Biochemical testing for neuroendocrine tumors. Pancreas 2009;38:876-889.

19. Bajetta E, Ferrari L, Martinetti A, et al. Chromogranin A, neuron specific enolase, carcinoembryonic antigen, and hydroxyindole acetic acid evaluation in patients with neuroendocrine tumors. Cancer 1999;86:858865.

20. Oberg K. Circulating biomarkers in gastroenteropancreatic neuroendocrine tumours. Endocr Relat Cancer 2011;18 Suppl 1:S17-S25.

21. Pereira PL, Roche AJ, Maier GW, et al. Insulinoma and islet cell hyperplasia: value of the calcium intraarterial stimulation test when findings of other preoperative studies are negative. Radiology 1998;206:703-709.

22. Paulson EK, McDermott VG, Keogan MT, DeLong DM, Frederick MG, Nelson RC. Carcinoid metastases to the liver: role of triple-phase helical CT. Radiology 1998;206:143-150.

23. Singhi AD, Chu LC, Tatsas AD, et al. Cystic pancreatic neuroendocrine tumors: a clinicopathologic study. Am J Surg Pathol 2012;36:1666-1673.

24. Sundin A. Radiological and nuclear medicine imaging of gastroenteropancreatic neuroendocrine tumours. Best Pract Res Clin Gastroenterol 2012;26:803-818.

25. Herwick S, Miller FH, Keppke AL. MRI of islet cell tumors of the pancreas. AJR Am J Roentgenol 2006;187:W472-W480.

26. Singh S, Law C. Chromogranin A: a sensitive biomarker for the detection and post-treatment monitoring of gastroenteropancreatic neuroendocrine tumors. Expert Rev Gastroenterol Hepatol 2012;6:313-334.

27. Dromain C, de Baere T, Baudin E, et al. MR imaging of hepatic metastases caused by neuroendocrine tumors: comparing four techniques. AJR Am J Roentgenol 2003;180:121-128.

28. Sundin A, Vullierme MP, Kaltsas G, Plöckinger U; Mallorca Consensus Conference participants; European Neuroendocrine Tumor Society. ENETS consensus guidelines for the standards of care in neuroendocrine tumors: radiological examinations. Neuroendocrinology 2009;90:167-183.

29. Dromain C, de Baere T, Lumbroso J, et al. Detection of liver metastases from endocrine tumors: a prospective comparison of somatostatin receptor scintigraphy, computed tomography, and magnetic resonance imaging. J Clin Oncol 2005;23:70-78.

30. Okabayashi T, Shima Y, Sumiyoshi T, et al. Diagnosis and management of insulinoma. World J Gastroenterol 2013;19:829-837.

31. Caramella C, Dromain C, De Baere T, et al. Endocrine pancreatic tumours: which are the most useful MRI sequences? Eur Radiol 2010;20:2618-2627.

32. Lamberts SW, Bakker WH, Reubi JC, Krenning EP. Somatostatin-receptor imaging in the localization of endocrine tumors. N Engl J Med
1990;323:1246-1249.

33. Fjällskog ML, Ludvigsen E, Stridsberg M, Oberg K, Eriksson B, Janson ET. Expression of somatostatin receptor subtypes 1 to 5 in tumor tissue and intratumoral vessels in malignant endocrine pancreatic tumors. Med Oncol 2003;20:59-67.

34. de Herder WW, Kwekkeboom DJ, Valkema R, et al. Neuroendocrine tumors and somatostatin: imaging techniques. J Endocrinol Invest 2005;28(11 Suppl International):132-136.

35. Zimmer T, Stölzel U, Bäder M, et al. Endoscopic ultrasonography and somatostatin receptor scintigraphy in the preoperative localisation of insulinomas and gastrinomas. Gut 1996;39:562-568.

36. Garin E, Le Jeune F, Devillers A, et al. Predictive value of 18F-FDG PET and somatostatin receptor scintigraphy in patients with metastatic endocrine tumors. J Nucl Med 2009;50:858-864.

37. Binderup T, Knigge U, Loft A, Federspiel B, Kjaer A. 18F-fluorodeoxyglucose positron emission tomography predicts survival of patients with neuroendocrine tumors. Clin Cancer Res 2010;16:978-985.

38. Buchmann I, Henze M, Engelbrecht S, et al. Comparison of 68Ga-DOTATOC PET and 111In-DTPAOC (Octreoscan) SPECT in patients with neuroendocrine tumours. Eur J Nucl Med Mol Imaging 2007;34:16171626.

39. Srirajaskanthan R, Kayani I, Quigley AM, Soh J, Caplin ME, Bomanji J. The role of $68 \mathrm{Ga}$-DOTATATE PET in patients with neuroendocrine tumors and negative or equivocal findings on 111In-DTPA-octreotide scintigraphy. J Nucl Med 2010;51:875-882.

40. Khashab MA, Yong E, Lennon AM, et al. EUS is still superior to multidetector computerized tomography for detection of pancreatic neuroendocrine tumors. Gastrointest Endosc 2011;73:691-696.

41. James PD, Tsolakis AV, Zhang M, et al. Incremental benefit of preoperative EUS for the detection of pancreatic neuroendocrine tumors: a meta-analysis. Gastrointest Endosc 2015;81:848-856.e1.

42. Leelasinjaroen P, Manatsathit W, Berri R, Barawi M, Gress FG. Role of preoperative endoscopic ultrasound-guided fine-needle tattooing of a pancreatic head insulinoma. World J Gastrointest Endosc 2014;6:506509.

43. Lennon AM, Newman N, Makary MA, et al. EUS-guided tattooing before laparoscopic distal pancreatic resection (with video). Gastrointest Endosc 2010;72:1089-1094.

44. Park DH, Choi JH, Oh D, et al. Endoscopic ultrasonography-guided ethanol ablation for small pancreatic neuroendocrine tumors: results of a pilot study. Clin Endosc 2015;48:158-164.

45. Han J, Chang KJ. Endoscopic ultrasound-guided direct intervention for solid pancreatic tumors. Clin Endosc 2017;50:126-137.

46. Braden B, Jenssen C, D’Onofrio M, et al. B-mode and contrast-enhancement characteristics of small nonincidental neuroendocrine pancreatic tumors. Endosc Ultrasound 2017;6:49-54.

47. Sakamoto H, Kitano M, Suetomi Y, Maekawa K, Takeyama Y, Kudo M. Utility of contrast-enhanced endoscopic ultrasonography for diagnosis of small pancreatic carcinomas. Ultrasound Med Biol 2008;34:525-532. 